\title{
Niveles de susceptibilidad a temefos en el vector transmisor del dengue en Trujillo, Perú
}

\author{
Julio Chávez ${ }^{1}$, Ofelia Córdova ${ }^{1}$, Franklin Vargas ${ }^{1}$
}

Resumen

\section{Dengue's transmitting vector susceptibility to temephos, Trujillo, Peru}

\begin{abstract}
Objective: To determine resistance levels to temephos in Aedes aegypti strains from Trujillo. Material and Methods: The larvae bioassays were done according to the World Health Organization's methodology. Third or early fourth stage larvae from each population were used, including the reference Rockefeller stock. Five replications from each insecticide concentration were done with mortalities varying between 2 and 98\%. Results: All studied strains were susceptible to temephos, with Florencia de Mora showing de highest resistance ratio with 3,05 $X$ value. Conclusion: Continuous resistance to temephos monitoring should be carried out in A. aegypti strains in order to decide on the correct use of this insecticide.
\end{abstract}

Key words: Aedes; dengue; temefos; biological assays.

\footnotetext{
1 Instituto de Investigación en Microbiología y Parasitología Tropical. Facultad de Ciencias Biológicas, Universidad Nacional de Trujillo. Trujillo, Perú.
}

\section{INTRODUCCIÓN}

Dentro del gran número de especies de mosquitos resistentes a la acción de insecticidas, se encuentra Aedes aegypti Linnaeus, que desempeña una importante función en la transmisión de enfermedades víricas. El dengue, fiebre del dengue hemorrágico y la fiebre amarilla son enfermedades transmitidas por esta especie que causan grandes impactos en salud pública $\left({ }^{1}\right)$.

El uso de insecticidas ha desempeñado un papel importante en los programas de control de $A$. aegypti. La alta resistencia a DDT (dicloro-difeniltricloretano) y otros insecticidas organoclorados llevó al uso de insecticidas organofosforados $\left({ }^{2}\right)$.

En el Perú, para el control de $A$. aegypti, el Ministerio de Salud ha hecho uso del larvicida organofosforado temefos $\left(^{3}\right)$. Recientemente, en el año 2000, este insecticida fue utilizado con alta intensidad cuando se presentaron los primeros casos de dengue en los distritos de la 
provincia de Trujillo, llegando a presentar un índice aédico del $23 \%$.

En el presente trabajo se hace una evaluación de los niveles de susceptibilidad a temefos en larvas de $A$. aegypti de 4 localidades de la Provincia de Trujillo, cuyos resultados aportarían información que puede contribuir al mejor uso de este insecticida para el control del vector del dengue.

\section{MATERIAL Y MÉTODOS}

Para el trabajo se utilizó poblaciones de Aedes aegypti de la Provincia Trujillo, específicamente de los distritos de La Esperanza, Florencia de Mora, Laredo, El Milagro y una cepa de referencia Rockefeller suministrada por el Instituto de Medicina Tropical "Pedro Kouri" (IPK), La Habana-Cuba. Se estableció y mantuvo las colonias en el insectario del Instituto de Investigación en Microbiología y Parasitología Tropical (INIMYPAT), con temperatura de $26 \pm 2{ }^{\circ} \mathrm{C}$ y humedad relativa de $65 \%$.

El insecticida temefos, al 93,3\% de pureza, fue suministrado por el Instituto de Medicina Tropical "Pedro Kouri”, La Habana-Cuba.

Se realizó los bioensayos siguiendo la metodología de la Organización Mundial de la Salud $\left({ }^{4}\right)$. Se empleó larvas del tercer o cuarto estadio temprano de cada población, incluyendo la cepa de referencia Rockefeller. Se usó 5 réplicas de cada concentración del insecticida (20 larvas por réplica) produciéndose mortalidades entre 2 y $98 \%$. Todas las soluciones fueron ajustadas a un volumen final de $1 \mathrm{~mL}$ con acetona. Esta concentración de acetona no causó mortalidad en los controles. La lectura de las mortalidades se realizó a las 24 h y los resultados fueron procesados mediante el programa probit-log $\left(^{5}\right)$ para obtener las concentraciones letales $\left(\mathrm{CL}_{50}\right)$.

El factor de resistencia $\left(\mathrm{FR}_{50}\right)$ fue calculado mediante la fórmula $\mathrm{FR}_{50}=\mathrm{CL}_{50}$ de la población
problema/CL $\mathrm{CL}_{50}$ de la población susceptible. Valores mayores de 5 indican resistencia y valores menores señalaría susceptibilidad $\left(^{6}\right)$.

\section{RESULTADOS}

Se encontró susceptibilidad a temefos en todas las poblaciones de Aedes aegypti estudiadas, al presentar valores de factor de resistencia menores de 5 .

Un mayor valor del factor de resistencia se encontró en la población de Florencia de Mora, con 3,05 X ,y menor valor en la población de El Milagro, con 2,19 X (Tabla 1).

Se observa que los valores de la pendiente van decreciendo a medida que aumenta el factor de resistencia, teniendo un menor valor la población de Florencia de Mora con 2,3 y mayor valor la población El Milagro con 3,4 (Tabla 2).

\section{DISCUSIÓN}

En el presente trabajo se determinó los niveles de resistencia a temefos en 4 distritos de la provincia de Trujillo. El nivel de susceptibilidad se demostró a través de los valores bajos y moderados del $\mathrm{FR}_{50}$ que presentaron las poblaciones estudiadas.

Durante los últimos 3 años, se ha venido controlando al vector Aedes aegypti con uso del organofosforado temefos, siendo el único larvicida utilizado por el Ministerio de Salud en la provincia de Trujillo. Ello indicaría que un uso constante de este único insecticida produciría una presión de selección, ocasionando la aparición de poblaciones resistentes, como se reporta para países como Cuba, Venezuela y Brasil, que presentaban factores de resistencia $89,91 \mathrm{X}, 11,1 \mathrm{X}$ y $15,5 \times{ }^{(6-8)}$.

En la Tabla 2 se aprecia un valor mayor de la pendiente en las poblaciones La Esperanza, El Milagro y la cepa Rockefeller, siendo estas 
Tabla 1. Concentraciones letales y factor de resistencia a temefos para larvas de Aedes aegypti en poblaciones de la provincia de Trujillo (La Libertad) comparado con la cepa de referencia Rockefeller.

\begin{tabular}{lcc}
\hline Procedencia & $\mathrm{CL}_{50}$ & $\mathrm{FR}_{50}$ \\
\hline Florencia de Mora & 0,03721 & 3,05 \\
Laredo & $(0,03324-0,03920)$ & \\
La Esperanza & 0,03440 & 2,82 \\
El Milagro & $0,03035-0,03736)$ & \\
& $(0,02598-0,03305)$ & 2,35 \\
Rockefeller* & 0,02671 & 2,19 \\
& $(0,02299-0,02878)$ & \\
& $(0,01178-0,01320)$ & \\
\hline
\end{tabular}

$C L_{50}$ : Concentraciones letales 50\%

$F R_{50}:$ Factor de resistencia $50 \%$

${ }^{*}$ Cepa de referencia

poblaciones más susceptibles a temefos que las poblaciones Laredo y Florencia de Mora, por lo que, cuanto más bajo sea el valor de la pendiente de la regresión, más heterogénea será la población y presentará una tendencia mayor a la resistencia $\left({ }^{6}\right)$.

Ha sido demostrada la correlación existente entre la actividad de esterasas detoxificativas y la resistencia a insecticidas organofosforados $\left({ }^{9,10}\right)$. En insectos, de los géneros Culex, Anopheles, Blatella y Pediculus, el mecanismo más común de resistencia a insecticidas organofosforados involucra la presencia de una o más esterasas $\left({ }^{11-13}\right)$, debido a una sobreproducción de las mismas, que tienen una alta afinidad por el enlace con el insecticida y teniendo una baja tasa de hidrólisis, principalmente actuando por secuestramiento del insecticida antes que éstos alcancen el blanco de la acetilcolinesterasa $\left({ }^{14}\right)$.

Los resultados obtenidos en el presente trabajo indican que el insecticida temefos puede seguir siendo usado en los programas de control vectorial del $A$. aegypti. A la vez, se hace necesario realizar monitoreos continuos de los
Tabla 2. Valor de la pendiente de regresión para poblaciones de Aedes aegypti de la provincia de Trujillo (La Libertad) comparado con la cepa de referencia Rockefeller.

\begin{tabular}{lc}
\hline Procedencia & $\beta$ \\
\hline Florencia de Mora & $2,3 \pm 0,25$ \\
Laredo & $2,8 \pm 0,33$ \\
La Esperanza & $3,1 \pm 0,55$ \\
El Milagro & $3,4 \pm 0,35$ \\
Rockefeller* & $4,5 \pm 0,29$ \\
\hline
\end{tabular}

${ }^{*}$ Cepa de referencia

niveles de resistencia en poblaciones de $A$. aegypti y así hacer un buen uso del temefos en el control de este vector.

\section{REFERENCIAS BIBLIOGRÁFICAS}

1. Lehane MJ. Biology of blood-sucking insects. London: Harper Collins Academia; 1991.

2. Rodríguez MM, Bisset JA, Díaz C, Soca L. Resistencia cruzada a piretroides en Aedes aegypti de Cuba inducido por la selección con el insecticida organofosforado malatión. Rev Cub Med Trop. 2003;55(2):105-11.

3. Polo JL. Vigilancia entomológica, epidemiológica y control vectorial del dengue en el sector Santo Toribio del distrito de Florencia de Mora, departamento La Libertad, abriljunio. Informe para obtener el Título Profesional de Biólogo. Universidad Nacional de Trujillo, Facultad de Ciencias Biológicas; 2000. 51 páginas.

4. World Health Organization. Instructions for determinig the susceptibility or resistance of mosquitoes larvae to insecticides. Unpublished document. WHO/VBC.81.807. 1981. p. 6.

5. Raymond M. Presentation d'une programme d'analyse logprobit pour microordinateur. Cahiers Orstrom. Series Entomology and Medical Parasitology. 1985;23:117-21.

6. Bisset JA, Rodríguez MM, Molina D, Díaz C, Soca LA. Esterasas elevadas como mecanismo de resistencia a insecticidas organofosforados en cepas de Aedes aegypti. Rev Cub Med Trop. 2001;53(1):37-43.

7. Braga IA, Pereira JB, Da Silva S, Valle D. Aedes aegypti resistance to temephos during 2001 in several municipalities in the states of Rio de Janeiro, Sergipe, and Alagoas, Brazil. Mem Inst Oswaldo Cruz. 2004;99(2):199-203.

8. Bisset JA, Rodríguez MM, Fernández D, Pérez O. Estado de la resistencia a insecticidas y mecanismos de resistencia 
en larvas del Municipio Playa, colectadas durante la etapa intensiva contra el Aedes aegypti en ciudad de La Habana, 2001-2002. Rev Cub Med Trop. 2004;56(1):61-6.

9. Callaghan A, Malcolm CA, Hemingway J. Biochemical studies of A and B carboxylesterases from organophosphates resistant strains of an Italian Culex pipiens (Diptera: Culicidae). Pest Bio Phys. 1991;41:198-206.

10. Pasteur N, Nancé E, Bons N. Tissue localization of overproduced esterases in the mosquito Culex pipiens (Diptera: Culicidae). J Med Entomol. 2001;38(6):791-801.

11. Amevigbe MD, Ferrer A, Champorie S, Monteny N, Deunff J, Lenoble DR. Isoenzymes of human lice: Pediculus humanus and Pediculus capitis. Med Vet Entomol. 2000;14:419-25.

12. Díaz C, Pérez M, Rodríguez MM, Calvo E, Bisset JA, Fresneda M. Resistencia a insecticidas en cepas de terreno de la especie Blatella germanica procedentes de Santiago de Cuba. Rev Cub Med Trop..2000;52(1):24-30.
13. Karunaratne SHPP, Hemingway J. Malathion resistance and prevalence of the malathion carboxylesterase mechanism in populations of mosquito vectors of disease in Sri Lanka. Bull World Health Organ. 2001;79(11):1060-4.

14. Cuany A, Handani J, Bergé J, Fournier D, Raymond M, Georghiou GP, Pasteur N. Action of esterase B1 on chlorpyrifos in organophosphate-resistant Culex mosquitoes. Pestic Biochem Physiol. 1993;45:1-6.

Manuscrito recibido el 15 de diciembre de 2004 y aceptado para publicación el 15 marzo 2005.

Correspondencia: Blgo. Julio César Chávez Galarza Instituto de Investigación en Microbiología y Parasitología Tropical, UNT Avda. EE UU 288 Urb. El Recreo. Trujillo, Perú

Correo-e: alucard_jul@hotmail.com 